\title{
Bemerkungen zu der Arbeit:
}

„Veber die Filtrationskraft des Bodens und die Fortschwemmang von

Bakterien durch das Grundwasser."

Versuche von Abba, Orlandi und Rondelli. ${ }^{1}$

Von

Prof. E. Pfuhl

in Berlin.

Zur Lösung der Frage, ob ein bereits vorhandener Brunnen, eine Quellfassung oder eine Sickergallerie Zuflüsse von verunreinigtem Grundwasser erhält, sind verschiedene Methoden ausgearbeitet worden. Eine zusammenfassende Uebersicht findet sich in der Arbeit von Gärtner: „Ueber Methoden, die Möglichkeit der Infection eines Wassers zu beurtheilen," welche in der Festschrift zur 100 jährigen Stiftungsfeier des Friedrich-Wilhelms-Institutes 1895 veröffentlicht worden ist. Ein von den üblichen Prüfungsmethoden abweichendes Verfahren habe ich seit dem Jahre 1895 bei Brunnen in solchen Fällen angewandt, wo ich wusste, dass die oberste Grundwasserschicht mit Bakterien verunreinigt war. Nachdem ich das Grundwasser an einer Stelle der Umgebung des Brunnens freigelegt hatte, mischte ich eine Prodigiosus-Bouilloncultur hinzu und prüfte, ob beim starken Pumpen der Prodigiosus in den Brunnen hineinsogen werden würde.

Zuerst übte ich dieses Verfahren bei meinen Laboratoriums-Untersuchungen ${ }^{2}$ über die Verunreinigung der Grundwasserbrannen von unten her, die ich im Jahre 1895 veröffentlicht habe. Noch in demselben Jahre konnte ich nachweisen, dass ein Abessinierbrunnen ${ }^{3}$ ein mit Prodigiosus ver-

${ }^{1}$ Diese Zeitschrift. Bd. XXXI.

2 Ebenda. Bd. XXI.

${ }^{3}$ Mitgetheilt in meiner Arbeit: Ueber die Verschleppung der Bakterien dureh das Grundwasser. Elenda. Bd. XXV.

Zoitschr, f, Hygiene, XXXI. 
unreinigtes Wasser gab, wenn ich $3.7^{\mathrm{m}}$ aufwärts die oberste Grundwassersehicht freilegte und eine Prodigiosuscultur beimischte. Abba, Orlandi und Rondelli haben nun im Jahre 1896, als sie die Filtrationskraft des Bodens oberhalb und zu beiden Seiten der Filtergallerie der Turiner Wasserleitung zu prüfen hatten, nicht erst das Grundwasser freigelegt, sondern die Prodigiosuscultur direct auf die Bodenoberfläche ausgegossen und gleichzeitig die Versuchsstelle überschwemmt. Dann haben sie darauf geachtet, ob der Prodigiosus mit den einsickernden Wässern bis in eine Filtergallerie gelangte. Wie sie angeben, sind dabei die Bakterien durch das Grundwasser bis zu $200^{\mathrm{m}}$ weit fortgeschwemmt worden. Diese Versuche sind meiner Meinung nach besonders deshalb lehrreich, weil sie zeigen, wie sehr die Sickergallerieen der Wasserleitungen', die mit Vorliebe im diluvialen Kies der Flussthäler angelegt werden, der Verunreinigung ausgesetzt sind, wenn sie überschwemmt werden, während bei andauernden Regengüssen die Gefahr der Verunreinigung sehr viel geringer ist. Ich glaube diese wichtige Schlussfolgerung besonders hervorheben $z \mathfrak{u}$ müssen, da die Verfasser in ihren Schlussfolgerungen hierauf nicht hingewiesen haben, sondern nur vom Einfluss der „,künstlich auf das Terrain der Gallerieen (zwecks Vermehrung des Wassers in denselben) geleiteten Wässer" sprechen. Dagegen sind die Verfasser auf meine im Jahre 1896 angestellten Versuche: „Ueber die Verschleppung von Bakterien durch das Grundwasser" näher eingegangen, die ich im Jahre 1897 in dieser Zeitschrift, Bd. XXV, veröffentlicht ${ }^{1}$ habe.

Da ich den Eindruck habe, als ob die Verfasser manches in meiner Arbeit übersehen haben, so halte ich es für angezeigt, mit einigen kurzen Bemerkungen die Sache klar zu stellen.

1 Abba, Orlandi und Rondelli haben über ihre Versuche schon im Juli 1896 einen Bericht in der Gazetia medica di Torino, Nr. 28 und darauf, wie sie selbst angeben, einen kurzen Auszug aus demselben „ebenfalls in demselben Jahre“, in Wirklichkeit aber im folgenden Jahre, im Centralblatt für Bakteriologie, Bd. XXI, S. 824, veröffentlicht. Als ich im Jahre 1897 meine Arbeit über die Verschleppung der Bakterien durch das Grundwasser veröffentlichte, kannte ich nur das Referat im Centralblatt für Bakteriologie und daraus auch den Titel der italienischen Originalarbeit: "Saggio di esperienze sul potere filtrante dei terreni." Weder aus dem Titel der Originalarbeit, noch aus dem Referat habe ich ersehen können, dass die Verfasser Versuche über die Verschleppung von Bakterien durch das Grundwasser angestellt hatten, denn das Referat handelte nur von den Versuchen über die Filtrationskraft des Bodens. Den Bericht über ihre Verschleppungsversuche haben sie den deutschen Lesern erst durch ihre Veröffentlichung in Bd. XXXI dieser Zeitschrift zugänglich gemacht. So ist es erklärlich, dass ich zur Zeit der Veröffentlichung meiner Arbeit ihre Verschleppungsversuche noch nicht kannte. 
Nachdem die Verfasser über meine im Jahre 1896 ausgeführten Untersuchungen berichtet haben, fahren sie fort: „dieselben Versuche machte er dann mit Abessinierbrunnen". Hiernach könnte es scheinen, als ob ich diese Versuche erst später angestellt hätte. Doch habe ich in meiner Arbeit ausdrücklich gesagt: „diese Versuche sind schon im Sommer 1895 ausgeführt". Sie sind also auch bereits vor den Untersuchungen der italienischen Forscher vorgenommen worden.

Am Schlusse der Mittheilung meiner Versuche sagen die Verfasser: „Wie man sieht, sind dieselben im Wesentlichen sogen. Laboratoriumsversuche, aus denen er selbst keine praktischen Schlüsse gezogen hat."

Warum meine Versuche, die ich am Grundwasser im gewachsenen Boden angestellt habe, als sogen. Laboratoriumsversuche anzusehen sind, ist mir nicht ohne Weiteres klar. Mit demselben Recht könnte man auch die Versuche der italienischen Forscher als sogen. Laboratoriumsversuche ansehen.

Was die Bemerkung anlangt, dass ich aus meinen Versuchen keine praktischen Schlüsse gezogen hätte, so möchte ich auf folgende Stelle in meiner Arbeit hinweisen: „Was für die leuchtenden Vibrionen gilt, dürfte auch für die Choleravibrionen zutreffen u. s. w." „In Folge dessen halte ich es für bedenklich, dass z. B. bei dem Sammelbrunnen eines Wasserwerkes, das ich im Elsass kennen gelernt habe, in $14^{\mathrm{m}}$ Entfernung ein Bach vorbeifliesst, der weiter oberhalb die Abwässer gedüngter Aecker aufnimmt."

Das sind doch Schlüsse, die auf die Praxis Rücksicht nehmen. Vielleicht haben die italienischen Forscher meine Versuche deshalb als Laboratoriumsversuche bezeichnet, weil sie es nicht für genügend begründet hielten, dass ich bei meinen Verschleppungsversuchen die Bakterien nicht auf die Bodenoberfläche, sondern in das freigelegte Grundwasser einsäte und den Grundwasserstrom durch Pumpen beschleunigte. Darauf deuten ihre Worte hin: „Wenn wir, wie Pfuhl es gethan hat, aufwärts von den Filtergallerieen eine Grube bis zur Freilegung des Wassers ausgeworfen, hier die Bakterien hineingesät und dann von den Gallerieen aus einen beschleunigten Zufluss von Wasser bewirkt hätten, so würde uns dies wie ein wenig Interesse bietender Versuch vorgekommen sein, denn wir würden uns in zu künstliche Verbältnisse gesetzt, wir würden einen zu directen Verbindungsweg geschaffen haben. Es schien uns, und derselben Ansicht sind wir auch heute noch, dass, wie sehr auch ein Grundwasserstrom pathogene Keime selbst auf grosse Strecken zu verschleppen vermag, dies an und für sich noch keinen schweren Uebelstand ausmache, wenn die Beschaffenheit des darüber gelegenen Bodens dessen Verunreinigung nicht gestattet." 
Hierauf habe ich zu erwidern, dass bereits aus den ersten Sätzen meiner Arbeit zu ersehen ist, weshalb ich ein anderes Verfahren eingeschlagen habe, als die italienischen Forscher. Diese Sätze lauten:

„Nachdem ich mich durch eigene Untersuchungen überzeugt hatte, dass an manchen Stellen der mittelrheinischen Ebene eine Verunreinigung der obersten Grundwasserschichten mit Bakterien vorkommt, unternahm ich es, festzustellen, ob und wieweit die in das Grundwasser hineingelangten Bakterien mit dem Grundwasserstrom weitergeschleppt werden kōnnen. Die Wichtigkeit dieser Frage hat man schon längst erkaunt. Hängt es doch von der Entscheidung derselben $a b$, ob eine Verunreinigung von Grundwasserbrunnen eintreten kann, wenn weiter aufwärts Bakterien in das Grundwasser hinein gelangen." Dies kann z. B. von undichten Senkgruben, Schwindgruben, undichten Canalisationsröhren und Gullies ans geschehen. Es wird doch Niemand leugnen, dass es von Interesse ist, zu erfahren, ob von solchen Stellen aus die in's Grundwasser gelangten Bakterien weiter fortgeschleppt werden können, da sich oft schon in geringer Entfernung Brunnen befinden, welche die Strömung des Grundwassers beschleunige n, wenn ihr Wasserspiegel durch Pumpen abgesenkt wird. Es entsprach deshalb gerade den wirklichen Verhältnissen, wenn ich bei meinen Versuchen die Bakterien direct der obersten Grundwasserschicht zusetzte und durch Pumpen eine schnellere Strömung des Grundwassers herbeiführte. Eine Beschleunigung des Grundwasserstromes ist übrigens auch bei den Versuchen der italienischen Forscher vorhanden gewesen, da doch das Wasser aus den Filtergallerieen fortwährend abfloss, beim 4. Versuch sogar ein grosser Theil des Wassers der rechten Gallerie, sowie das der linken Gallerie abgelassen wurde. Durch das Ablaufen des Wassers musste doch eine Absenkung des Grundwasserspiegels und damit eine schnellere Zuströmung des Grundwassers von den Seiten her nach der Filtergallerie erfolgen. Ausserdem beschleunigten die Verfasser noch dadurch die Fortschwemmung der Bakterien, dass sie das Versuchsgelände bis $10^{\mathrm{cm}}$ hoch überschwemmten.

Was die absprechenden Bemerkungen dèr 3 Forscher über meine Empfehlung des leuchtenden Vibrio anbetrifft, so erscheint es mir fraglich, ob sie auf Grund eigener Versuche zu diesem Urtheil gekommen sind.

Wenn die Verfasser glauben, dass sie für den Nachweis einer Verunreinigung der Filtergallerieen von der 0 berfläche des Quellengebietes den einzuschlagenden Weg angegeben haben, so möchte ich darauf hinweisen, dass man schon früher andere Wege kannte, wie ich am Anfang dieser Arbeit angegeben habe. Man braucht z. B. nur neben den Filtergallerieen Abessinierbrunnen einzuschlagen, um nach der Desinfection 
Bemerkungen zur Filltrationskraft des Bodens U. s. w. 501

derselben festzustellen, ob das zu den Gallerieen strömende Grundwasser Bakterien enthält oder nicht.

Das letztere Verfahren ist fast immer anwendbar, auch im diluvialen Kiesboden der Flussthäler, während das Verfahren der italienischen Forscher eigentlich nur für die Fälle passt, wo die Gefahr einer Verunrei nigung durch Ueberschwemmung in Frage kommt. Doch erscheint es fraglich, ob die Finwohner der betreffenden Stadt die Verunreinigung ihrer Wasserleitung mit Prodigiosus und anderen Bakterien sich während der Versuche werden gefallen lassen. Jedenfalls müsste dafür gesorgt werden, dass nicht neben dem Prodigiosus noch infectiöse Bakterien in die Wasserleitung gelangen.

Das von mir benutzte Verfahren ist besonders für die Fälle geeignet, wo man sich darüber unterrichten will, wie weit in einem Boden von bestimmter Beschaffenheit die Bakterien durch das Grundwasser fortgeschleppt werden können. Dies kann z. B. dann in Frage kommen, wenn bestimmt werden soll, wie weit von einer schon vorhandenen Senkgrube oder von der Grenze des Nachbargrundstückes entfernt ein neuer Brunnen angelegt werden soll. Der Versuch braucht dann gar nicht einmal an derselben Stelle vorgenommen zu werden, wo nachher der Brunnen gebaut werden soll, sondern kann auch in einiger Entfernung an einer geeigneten Stelle stattfinden, wo der Boden von der gleichen Besehaffenheit ist. 